Projets

de paysage

\section{Projets de paysage}

Revue scientifique sur la conception et l'aménagement de l'espace

9 | 2013

Le paysage a t-il imposé sa loi ?

\title{
Perspectives paysagères. La recherche en questions
}

À propos des cinquièmes Journées doctorales en paysage de Bordeaux (13 au 15 décembre 2012)

Landscape perspectives : research issues - Concerning the Fifth Bordeaux

Landscape Doctoral Days (13-15 December, 2012)

\section{Georges Bertrand}

\section{(2) OpenEdition}

Journals

Édition électronique

URL : http://journals.openedition.org/paysage/13033

DOI : $10.4000 /$ paysage. 13033

ISSN : 1969-6124

Éditeur :

École nationale supérieure du paysage de Versailles-Marseille, Institut national des sciences appliquées Centre Val de Loire - École de la nature et du paysage, École nationale supérieure d'architecture et de paysage de Bordeaux, École nationale supérieure d'architecture et de paysage de Lille, Agrocampus Angers

\section{Référence électronique}

Georges Bertrand, «Perspectives paysagères. La recherche en questions », Projets de paysage [En ligne], 9 | 2013, mis en ligne le 20 décembre 2013, consulté le 18 novembre 2020. URL : http:// journals.openedition.org/paysage/13033; DOI : https://doi.org/10.4000/paysage.13033

Ce document a été généré automatiquement le 18 novembre 2020.

Projets de paysage 


\title{
Perspectives paysagères. La recherche en questions
}

\author{
À propos des cinquièmes Journées doctorales en paysage de Bordeaux \\ (13 au 15 décembre 2012) \\ Landscape perspectives : research issues - Concerning the Fifth Bordeaux \\ Landscape Doctoral Days (13-15 December, 2012)
}

\section{Georges Bertrand}

1 Serge Briffaud, organisateur de ces Journées doctorales en paysage, m'a proposé de rédiger une sorte de "postface», ce qui ne veut rien dire... ou tout dire s'il s'agit de ménager un espace de libre réflexion à partir des travaux présentés et, si possible, d'élargir le panorama. Une occasion unique de mettre des recherches paysagères récentes en perspective générale. En effet, ce ne peut être un bilan: celui-ci relève collectivement du conseil scientifique de cette manifestation. Une analyse critique serait elle aussi déplacée dans la mesure où les intervenants sont des chercheurs débutants, la plupart encore doctorants, qui ont vaillamment accepté de présenter leurs réflexions et leurs travaux, pour certains encore en pleine période d'investigation et d'interrogation. De leur part il y a une attente. Il faut y répondre en prolongeant leurs questionnements.

2 Des écoutes, malheureusement intermittentes, et des échanges, publics ou en aparté, constituent la matière première de cette mise en perspective de recherches que les doctorants d'aujourd'hui auront demain la charge de mettre en pratique.

\section{"La méthode avant toute chose »}

3 Aborder la question du paysage par la recherche, en insistant tout particulièrement sur la méthode, n'est pas chose commune. L'initiative de ces Journées doctorales doit être saluée, d'autant qu'elle intervient à une période décisive pour le paysage. Les études paysagères entrent, à leur tour, dans la crise générale sociale et environnementale qui va exiger, avec une économie de moyens, de plus en plus de rigueur et d'innovation. Le 
paysage, tel qu'en lui-même, peut en être la pire (superfétatoire, discoureuse...) ou la meilleure (combinatoire, humaniste...) des méthodes de connaissance et d'action.

\section{« Il faut s'attendre à l'inattendu »: quel paysage pour temps de crise?}

Si Edgar Morin donne du sens et un sens, il ne montre pas la voie. Entre la culture et l'environnement, l'art et le territoire, il n'y a pas de chemin tout tracé dans les paysages de demain. Dans son développement actuel, le paysage peut être globalement considéré comme un fruit tardif des Trente Glorieuses, période faste et insoucieuse où les idées de progrès, de culture et de sensibilité avaient encore un bel avenir avec le paysage comme mise en scène ou fond de décor.

La crise contemporaine n'efface pas la recherche paysagère : elle la repositionne et la responsabilise. Quel paysage pour temps de crise ? Un paysage lui-même menacé par la conjonction d'une urbanisation accélérée et d'une crise climatique et environnementale généralisée ? Un paysage comme supplétif «sensible » et simple fond de tableau ou bien, au contraire, un paysage conceptualisé, fédérateur de configurations culturelles et territoriales inédites ? À l'exemple de l'écosystème qui a rassemblé dans les décennies 1960-1980 le monde vivant et joué un rôle déterminant dans l'émergence de la pensée environnementale ? Pour prétendre accéder à une telle révolution conceptuelle le paysage doit, sans renoncer à son socle culturel, patrimonial et sensible, s'ouvrir à d'autres dimensions écologiques, économiques et sociales, en particulier dans le domaine du développement des territoires. De fait, le paysage est entièrement à repositionner dans la complexité et la diversité du monde contemporain. Une stratégie irréalisable sans changement de méthode, c'est-à-dire sans redéfinition de la recherche paysagère.

\section{La recherche, maillon faible des études paysagères}

6 Le paysage demeure encore un objet-sujet scientifique mal identifié et mal traité. Les Journées doctorales de Bordeaux le confirment. Pratiquement toutes les études démarrent comme si le paysage, en se fiant à ses seules apparences, allait de soi. Une interdisciplinarité à tous vents est appelée à la rescousse. Or l'individualisation du paysage ne peut se construire à partir de «l'amoncellement sans liens des connaissances qui produit une nouvelle et très docte ignorance chez les experts et les spécialistes prétendant éclairer les responsables politiques et sociaux ${ }^{1}$ ». La complexitédiversité qui fonde la notion de paysage, qui plus est à la charnière culture-nature, nécessite une conceptualisation ad hoc qui n'a pas d'équivalent connu dans le domaine scientifique. Elle implique un métissage qui transcende les théories et les méthodes disciplinaires et interroge la traditionnelle rationalité scientifique. Des pistes existent. Par exemple, l'hypothèse de tester sur le paysage les principes épistémologiques et méthodologiques de l'analyse de système, en prévoyant plusieurs entrées et sorties dans le même objet-sujet paysage (ou territoire). Le système GTP en est une première expérimentation. Cette indispensable construction scientifique ne doit pas écarter la dimension descriptive, littéraire et poétique, qui reste l'irréductible apanage de l'analyse paysagère. De ce point de vue, l'iconographie sous toutes ses formes, largement et heureusement utilisée pendant ces Journées, constitue une dimension de 
la méthode, à condition qu'elle soit raisonnée sans rien perdre des couleurs et de la chaleur du paysage. Entre matérialité et idéalité il reste toute une approche méthodologique à repenser. Le paysagisme y joue une crédibilité qu'il est peut-être en train de perdre.

7 Enfin devenue une dimension incontournable des politiques territoriales publiques ou privées la recherche paysagère devra se renforcer du côté des méthodes et techniques de gestion, administrative et juridique.

\section{Questions pour une prospective paysagère}

8 On ne peut pas reprocher à des chercheurs-doctorants de se cantonner à de sages monographies. C'est la loi du genre. Une pratique qui favorise davantage la reproduction sociale et scientifique que l'imagination créatrice. Toutefois, même dans ce cadrage académique, la plupart des études présentées pendant ces Journées s'efforcent d'innover sinon les problématiques du moins les thématiques, de la ville nouvelle au patrimoine naturel, de l'économique à l'affectif, du jardin clos à l'infini de l'horizon. Autant de problèmes concrets qui pèseront sur l'avenir des études paysagères et, plus prosaïquement, sur le devenir professionnel des jeunes chercheurs. Nous esquissons ici, à grands traits et sous forme de questions vives, quelques-unes de ces pistes évoquées avec les différents participants.

\section{Assumer le nœud gordien entre environnement et paysage ?}

9 C'est la question fondamentale, toujours latente, et la grande ambiguïté des études paysagères. On a cru, un temps, éluder le problème en isolant artificiellement le paysage de toute contamination écologique et environnementale, puis en bricolant quelques passerelles. Les travaux présentés au cours de ces Journées souffrent de ces incohérences et malentendus.

10 Le problème est à reprendre à la base car, faute d'une culture historique et épistémologique suffisante, les chercheurs n'ont pas la capacité critique d'évaluer une situation jusqu'ici inédite : d'une part, du fait du rapprochement de deux champs, l'un culturel, l'autre naturaliste, qui se sont longtemps ignorés et qui ont beaucoup de difficultés à communiquer de part et d'autre de la diacrisis nature-culture; d'autre part, du fait de la convergence confuse et brutale de ces deux approches sur une problématique territoriale très gestionnaire.

11 Au-delà des corporatismes et des pratiques convenues, peut-être par-delà même le paysage et l'environnement, faut-il imaginer et mettre en œuvre de nouveaux paradigmes associant la culture et la science, l'épistémologie et la méthode, le rationnel et le sensible? Un basculement du paysage du côté de l'environnement et du territoire qui pourrait être révolutionnaire et dont les Journées doctorales de Bordeaux préfigureraient une première étape? 


\section{Réduire l'écart grandissant entre la grande culture historique du paysage et les pratiques territoriales ?}

Les références à la longue et riche histoire du paysage se font rares ou de pure convention. L'artialisation, par exemple, n'est pour beaucoup de jeunes chercheurs qu'un pieux souvenir. Les théories paysagères ne sont plus que des fils ténus qui tendent à se rompre. L'action immédiate accapare l'attention et les moyens. La priorité va à la présentation de résultats "concrets", c'est-à-dire économiques et environnementaux; l'esthétique est subordonnée. La recherche paysagère s'instrumentalise et la recherche fondamentale est délaissée. Seules émergent des représentations socioculturelles confinées à l'immédiateté du sensible et à une patrimonialisation réduite à une dimension parmi d'autres de l'aménagement. Que le paysage soit devenu un outil territorial est une chose tout à fait positive et riche de possibilités mais n'a-t-il pas perdu la saveur et les couleurs de ses origines ? La lecture de beaucoup de projets paysagers ici présentés n'est souvent que la traversée d'une morne plaine. Alors que le paysage devient une mode et une nécessité, la culture paysagère, faute d'entretien historique, s'affadit et menace de s'estomper. C'est un enjeu majeur pour la formation contemporaine, pas seulement dans les écoles de paysage mais partout où il est question d'aménagement-développement des territoires.

\section{Dépasser des interdisciplinarités de circonstance?}

13 Les études paysagères contemporaines reposent, pratiquement sans exception, sur de très larges interdisciplinarités, tout particulièrement entre sciences de la société et sciences de la nature. Les Journées doctorales en sont une illustration probante. Non seulement les chercheurs appartiennent à différentes disciplines mais encore ils en manipulent plus ou moins les méthodes et les techniques. Par contre, paradoxe académique, les travaux restent individuels, chacun sur son quant-à-soi monographique.

Faut-il se satisfaire de cette forme assez efficace mais quelque peu bancale d'interdisciplinarité, ou bien faut-il y voir une étape transitoire vers des méthodologies plus construites et plus spécifiques? L'idée d'une "science du paysage", autonome, voire indépendante, formulée dès les années 1970, a depuis été jugée irréaliste. Ne fautil pas en reprendre aujourd'hui les principes épistémologiques et méthodologiques sur des bases plus assurées pour donner un "corps » au paysage ? Le modèle étant celui d'une «science diagonale " au sens du philosophe Roger Caillois. C'est une belle utopie. Elle est en marche. Il ne faut pas l'arrêter. Le résultat est imprévisible mais le cheminement pour y parvenir, plein de seuils et de bifurcations, est riche d'enseignements. Le paysage, par sa superficialité et ses approximations méthodologiques, ne doit plus être considéré comme une facilité pratique, à l'écart des disciplines et des interdisciplines de l'environnement et du développement.

\section{Ouvrir la porte du jardin sur un territoire pluridimensionnel ?}

15 Le jardin a été le terreau dans lequel le paysage contemporain a pris racine et s'est épanoui. Une référence symbolique pour tous mais qui ne peut plus être absolue. Le jardin n'est que la plus petite partie d'un tout qui le dépasse. Extraordinaire ou 
ordinaire le paysage exprime aujourd'hui, sous ces multiples facettes matérielles et symboliques, la globalité et la continuité de l'espace vécu dont il est devenu, par là même, un instrument d'analyse et de gestion. Un changement d'échelle qui est un changement de nature et de finalité, bien au-delà des pratiques jardinatoires et de l'art topiaire. Les méthodes n'ont pas suivi et les résultats en pâtissent. Les travaux présentés aux Journées doctorales se situent dans cet entre-deux incertain et louvoient parmi des interdisciplinarités approximatives. Le repli sur les méthodes monographiques d'inspiration géographique est une facilité. Il gomme la spécificité culturelle et sensible du paysage au profit d'une conception environnementaliste, en retrait par rapport à certaines dimensions récentes du paysagisme. Les jeunes chercheurs doivent revisiter leur jardin et imaginer une opération « portes ouvertes » sur de plus grands paysages, en particulier ceux des pays du Sud.

\section{Acculturer la nature au travers du paysage?}

Discrètement évoquée, la nature n'est jamais totalement absente des communications, même lorsqu'il s'agit de paysages urbains artificialisés. Mais de quelle nature s'agit-il, biophysique et/ou transcendantale? Quelle place et quel rôle lui attribuer dans la combinaison paysagère ? Il y a là une erreur de perspective et, plus gravement encore, de prospective. Paradoxalement, le paysage participe d'un retour à la nature (renouveau d'une pensée et d'un "sentiment de la nature " aussi bien dans le vécu quotidien que dans les expressions artistiques) et d'un retour de la nature (crise environnementale et crise climatique, voire "catastrophe»). L'anthropisation, l'artificialisation tous azimuts, voire l'artialisation, ont redonné une seconde vie à une naturalité comme dimension intrinsèque du paysage. La nature «naturaliste » est en quelque sorte acculturée au travers du paysage par une pensée métisse qui transcende la coupure nature/culture. C'est l'une des grandes acquisitions de la recherche paysagère. La méthode, mixte par définition, doit en rendre compte. Il n'y a pas de paysage sans nature et il ne peut y avoir de méthode paysagère autrement qu'à la charnière entre sciences sociales et sciences de la nature, soit une culture scientifique encore à improviser. Une nature que les jeunes chercheurs des Journées ont, semble-til, beaucoup de mal à aborder mais qu'ils n'ignorent pas.

\section{Sortir de l'européocentrisme et de l'ineptie des « sociétés sans paysage »}

17 Dès leur introduction, les Journées doctorales de Bordeaux ont brillamment élargi la «fabrique du paysage » à des sociétés et à des paysages hors d'Europe (Cameroun). Les "sociétés sans paysage » sont définitivement à ranger sur le même rayon poussiéreux que les trop fameuses « sociétés sans histoire ». La traditionnelle conception très (trop) intellectualisée et très (trop) européocentrée, élitiste et ésotérique, est culturellement et scientifiquement dépassée comme relents colonialiste et impérialiste. Il était temps que les recherches sur le paysage intègrent ce que disent depuis fort longtemps les ethnologues et les anthropologues (Claude Lévi-Strauss, Philippe Descola...).

La première condition est de dépasser les principes «circulaires" de la Convention européenne du paysage pour retrouver un paysage plus universel dans sa diversité culturelle et territoriale. 
19 La deuxième condition est de contrebalancer l'influence devenue universelle d'une pensée écologique et environnementale, vecteur d' «ingérences écologiques » qui menace les sociétés et les paysages des pays du Sud. Une fonction humaniste que le paysage doit assumer à la condition de s'immerger dans les cultures et les sociétés locales en dépassant les constructions paysagères européennes.

\section{Les malentendus de la médiation}

20 La médiation est à la mode chez les paysagistes comme dans toute la société. Elle a été largement évoquée tout au long de ces Journées. À bon escient et à moins bon escient, sans que l'on sache ce qu'il en est réellement des pratiques. L'ambiguïté est totale. Il faut effectivement distinguer ce qui revient à la médiation interne au paysage et ce qui relève des procédures d'aménagement.

$21 \mathrm{Du}$ point de vue interne, la médiation est une démarche conceptuelle et méthodologique essentielle. Le paysage est en lui-même un objet de médiation tout à fait exceptionnel : culture-nature, matériel-idéel, objectif-subjectif, etc. On peut parler d'une indispensable et constante "médiation constitutive». Elle permet de surmonter la complexité-diversité inhérente au paysage en dynamisant les interfaces et en colmatant les interstices. Dans la méthode, cela se traduit par une médiation constante (et pas toujours réussie) entre, d'une part, les sciences de la société et les sciences physiques, et d'autre part, entre la recherche fondamentale et ses applications. Cette forme de médiation est généralement négligée par l'ensemble des chercheurs.

22 La médiation externe, sociale, est d'une tout autre nature et exigence. D'abord, elle ne peut à elle seule recouvrir toutes les formes d'intervention sur le territoire et sa gestion. Elle correspond à une fonction précise. Elle est destinée à mettre d'accord, à concilier ou à réconcilier des acteurs, à servir d'intermédiaire pour régler un conflit (Petit Robert). Le médiateur est un facilitateur, donc au-dessus de la mêlée, alors que le chercheur, le plus souvent en position contractuelle, ne peut être considéré comme totalement indépendant des parties en conflit. D'où le peu d'efficacité de ses recommandations et de son projet. Certes, le paysagiste, généraliste par définition, n'est pas le plus mal placé pour établir un tel dialogue. Encore faut-il qu'il ait les capacités et la formation pour remplir une telle fonction. Le chercheur n'est pas, naturellement, un médiateur. La médiation se situe dans le prolongement normal de la recherche mais elle en diffère par ses connaissances et ses pratiques, c'est-à-dire par une méthode spécifique. Il n'y a pas de place pour l'improvisation. La médiation ne doit pas devenir un miroir aux alouettes pour des doctorants paysagistes qui en attendent trop sans avoir la formation nécessaire pour l'assumer. Enfin, il y a bien d'autres interventions pratiques pour le paysagiste.

\section{Ouverture de pistes}

23 Les cinquièmes Journées doctorales de Bordeaux ont permis à des doctorants et à de jeunes docteurs d'aborder avec pertinence des questions nouvelles, sinon bien renouvelées, et d'apporter un vif élan à des recherches paysagères somme toute classiques. Il faudrait signaler ici quelques thématiques novatrices concernant les affects paysagers, les paysages de l'énergie en montagne, la ville-nature, le paysage écologisé, le multisensoriel paysager, etc. Remarquons ici, à titre illustratif, deux 
brillantes communications qui ne peuvent que renforcer l'efficience et la crédibilité des analyses paysagères comparées à d'autres démarches de type environnemental.

\section{Le paysage fabrique d'oubli}

24 On a beaucoup travaillé sur la connaissance de l'histoire longue du paysage, son continuum patrimonial et sur ses rapports compliqués avec des mémoires successives et sélectives. Or, la mémoire ne va pas sans oubli et l'oubli contribue à façonner le paysage. Du palimpseste paysager on a surtout retenu ce qui a été sauvegardé et on a trop souvent négligé les ratures et les grattages, tout ce qui a été effacé, les discontinuités et la part de l'indéchiffrable. Les géologues ont en grande partie bâti l'histoire de la Terre sur les lacunes stratigraphiques qui correspondent presque toujours à des étapes décisives de l'évolution. Il n'y a pas de paysage sans oubli et chaque lacune a sa signification qui retentit sur l'interprétation des paysages contemporains et leur valorisation. Nous payons aujourd'hui l'oubli massif du paysage dans l'aménagement du territoire pendant les Trente Glorieuses. Quels oublis fabrique$\mathrm{t}$-on pour demain dans les paysages d'aujourd'hui?

\section{La mise en horizon du territoire}

Nous voici, enfin, de plain-pied dans le paysage tel que nous le voyons et le vivons. Un spectacle dressé comme un décor, construit comme une mise en scène, animé comme une pièce de théâtre. Dans le jeu des ciels et des nuages, des ombres et des lumières, dans le glissement des échappées et des masques avec, entre terre et ciel, cette ligne d'horizon qui dresse le paysage à hauteur d'homme. Faire de l'horizon une manière et une " matière à habiter le paysage » souligne la démarche rigoureuse de l'architecte qui élargit son propos à l'horizon paysager mobile. Cette mise en horizon est fondatrice. Elle distingue définitivement le paysage de l'environnement et des autres notions ou concepts territoriaux et donne au paysage un rôle spécifique dans les projets de développement et d'aménagement. Il est l'horizon de chacun.

Les cinquièmes Journées doctorales en paysage de Bordeaux (décembre 2012) se sont terminées en haut du clocher de Saint-Émilion, dans une bise aigre et sous un ciel tourmenté percé de rares rais de lumière. Au-dessus des toitures et des murailles surgies du tréfonds de la dalle calcaire. Face au puissant panorama des vignes méticuleusement encloses, château Ausone au premier plan, lointaines prairies bouclées par la Dordogne. Un extraordinaire paysage dans son ordinaire de paysage de terre et de ciel, de culture et de nature, sa charge d'histoire et de mémoire, son dynamisme social et économique de plus en plus tourné vers la Chine et vers le monde. Loin de la « docte ignorance » stigmatisée par Edgar Morin, dans le droit fil de Journées doctorales d'un très bon cru.

Toulouse, décembre 2012 - janvier 2013 


\section{NOTES}

1. Morin, E., Le Monde, 2 janvier 2013.

\section{INDEX}

Keywords : landscape research, Landscape doctoral days, landscape analysis, environment and climate crisis, mediation

Mots-clés : recherche paysagère, Journées doctorales en paysage, analyse paysagère, crise environnementale et climatique, médiation

\section{AUTEUR}

\section{GEORGES BERTRAND}

Géographe français, spécialiste de l'environnement et du paysage, Georges Bertrand a contribué à l'émergence d'une école toulousaine de géographie en refondant les concepts et méthodes de la discipline. Il est aujourd'hui professeur émérite de géographie à l'université de Toulouse II - Le Mirail, directeur de l'Institut toulousain du paysage et membre d'honneur de la Société catalane de géographie.

gbertran[at]univ-tlse2[dot]fr 